\title{
Development and Implementation of High Precision Positioning Technology for Electric Vehicle Wireless Charging Coils
}

\author{
Jie LI ${ }^{\mathrm{a}}$, Kejun QIAN ${ }^{\mathrm{a}}$, Yafei LI ${ }^{\mathrm{a}}$, Yi LIU ${ }^{\mathrm{a}}$, Zifeng LIU $^{\mathrm{b}}{ }^{1}$, Linlin TAN ${ }^{\mathrm{b}}$ \\ a Jiangsu Electric Power Company Suzhou Power Supply Company, Suzhou, China \\ ${ }^{b}$ School of Electrical Engineering, Southeast University, Nanjing, China
}

\begin{abstract}
This paper mainly studies the development and implementation of the positioning technology of the electric vehicle wireless charging coil, so as to accurately detect the position deviation of the receiving coil, so that the electric vehicle wireless charging system can provide electric energy for electric vehicles more efficiently. Based on the positioning method of electric vehicle based on three detection coils, this paper proposes a calculation method to describe the offset degree of coil based on fuzzy mathematics theory. The algorithm is verified by simulation and experiment, and the influence factors of the error accuracy and the source of the error are analyzed. The work done in this paper has a strong practical significance for the efficient realization of electric vehicle wireless energy transmission.
\end{abstract}

Keywords. electric vehicle wireless charging, three detection coils, fuzzy mathematics theory, coil offset

\section{Introduction}

According to a report by Marketsand-Markets, a market research and consulting firm, by 2020 , wireless charging for electric vehicles has generated a staggering $\$ 13.78$ billion in revenue worldwide [1]. At present, it seems that EV charging technology still has many technical bottlenecks: Compared with traditional cars, the charging duration and range of ELECTRIC vehicles are urgent problems to be solved, and there is no mature technical scheme for high-power charging either at the end of the power grid or at the end of electric vehicles. The huge gap with traditional vehicles in the number and scale of gas stations and other infrastructure is the bottleneck that electric vehicles must overcome. Charging facilities need to be built reasonably and effectively to overcome the range anxiety of electric vehicles.[2] Therefore, how to achieve efficient charging is now one of the core issues attracting much attention. Wireless charging of electric vehicles is non-

${ }^{1}$ Corresponding author: Zifeng Liu, School of Electrical Engineering, Southeast University, Nanjing, People's Republic of China; E-mail: 1282402375@qq.com. 
contact charging, so the charging coil can be buried underground to save space. Another advantage is that it is convenient to fix the transmitting coil underground to prevent unnecessary position shift of the transmitting coil due to various factors. The receiving coil can be fixed on the chassis of the electric vehicle. The electric energy is transmitted through the electromagnetic coupling between the transmitting coil and the receiving coil. In general, the transmitting coil of wireless charging is placed underground, and in some special cases, it can be placed on the ground, provided that strict height calibration is required [3]. The premise of efficient charging is the need to achieve accurate alignment between the transmitting coil and the receiving coil.

The mismatch caused by coil offset will have a significant impact on the transmission efficiency of electric energy, and may lead to the instability of resonant wireless energy transmission system. When the coil mismatch increases, the mutual inductance value between induction coils will decline sharply. The coils need to be nearly perfectly aligned to keep the power transmitted wirelessly within an acceptable range. Generally, the maximum allowable offset of the radio energy transmission system is $10 \mathrm{~cm}$, and the power transmission efficiency within the offset range at this position is within the acceptable range. With the increase of the offset, the transmission efficiency will decline sharply. However, some studies show that most drivers cannot accurately stop within the error allowable range. In addition, the average offset of parking will exceed $70 \mathrm{~cm}$ when the driver has no knowledge of any external device [4]. Therefore, the offset of the coil needs to be detected and corrected to maintain acceptable power transfer efficiency.

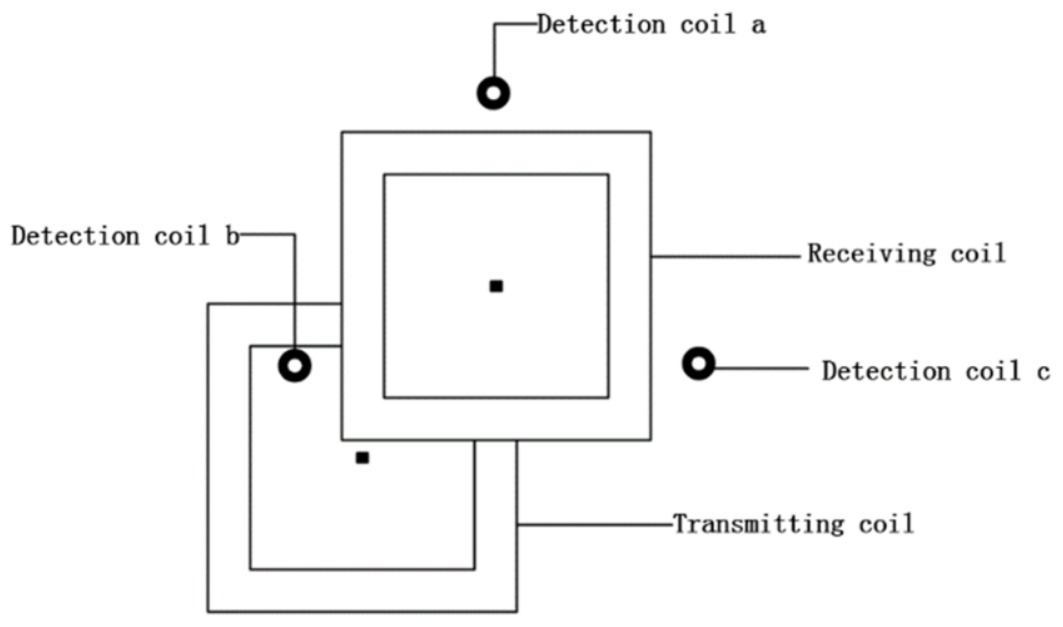

Figure 1. Schematic diagram of balance coil positioning scheme system.

The electric vehicle charging positioning has a lot of the existing research results, China first automobile limited liability company is proposed to increase the four testing coil, to distinguish the ground end and on-board the relative position [5]. Huazhong University of Science and Technology proposed a method of coupling the active excitation of secondary side coil with primary side coil to realize real-time position detection of vehicles by measuring the current variation in primary side coil [6]. Zhejiang University proposed an online wireless charging positioning system for electric vehicles, which made the output voltage on the differential inductor coil by coupling the secondary 
power coil after energization, and then obtained the relationship table between the output voltage of the differential inductor coil and the position of the secondary coil through a large number of experiments, so as to determine the position of the vehicle [7]. A position detection scheme for dynamic wireless charging system of electric vehicles based on magnetic positioning technology was designed in Beijing Jiaotong University [8]. Allan et al. from the International Society of Automotive Engineers have proposed a method for detecting the position of charging target devices in parking lot scenarios by evaluating the received signal strength indication of dedicated short-range communication signals and global positioning system data [9].

The main work content of this paper is to investigate the existing methods of electric vehicle wireless charging positioning, introduce the working principle of electric vehicle wireless charging positioning system and expatiate on its research significance. Analyze the electromagnetic model and circuit model of wireless charging coil positioning system, analyze the models of transmitting coil, receiving coil and detecting coil in the wireless charging system, design the parameters of detecting coil, and analyze the LCC-S circuit model of the wireless energy transmission system. The theoretical feasibility of wireless charging positioning method based on three detection coils is verified. Figure 1 shows the schematic diagram of balance coil positioning scheme system.

On the basis of the positioning method of electric vehicle based on three detection coils proposed in this paper, a calculation method based on fuzzy mathematics theory is put forward to describe the offset degree of the coil. The algorithm is verified by simulation, and the simulation results verify the effectiveness of the proposed method. The simulation results show that the smaller the mesh width is, the higher the positioning accuracy is. Then the influence factors of error precision and the source of error are analyzed.

\section{Characteristic Analysis of Electric Vehicle Wireless Charging Coil System}

\subsection{Analysis of Basic Model of Coupling Coil}

The energy transmission of the electric vehicle wireless energy transmission system is realized by the electromagnetic coupling between the receiving coil and the transmitting coil. The efficiency of the system's energy transmission is closely related to the coupling coefficient between the coils. In this chapter, the coupling coil model is firstly analyzed, and then the resonant compensation network LCC-S circuit model is analyzed. ANSYS Maxwell electromagnetic simulation software was used for auxiliary calculation.

\subsubsection{Analysis of Basic Electromagnetic Model of Rectangular Coil}

The mutual inductance between rectangular coils has a great influence on the transmission efficiency of electric vehicle wireless charging system. For the mutual inductance between them, Niemann formula can be used for derivation. The Niemann formula is as follows:

$$
M=\frac{\mu_{0}}{4 \pi} \oint p \oint a \frac{d \bar{p} d \vec{a}}{r}
$$

Figure. 2 shows the schematic diagram of mutual inductance calculation of rectangular coil: 
In the figure, coil 1 represents the transmitting coil, and coil 2 represents the receiving coil. It can be seen that the transmitting coil $\llbracket \mathrm{O} \rrbracket$ 1 is located at the origin of the spatial coordinates, and the coordinate of the receiving coil O_2 is ( $\mathrm{p}, \mathrm{q}, \mathrm{h})$, The two sides of the transmitting coil and the receiving coil can be named as ABCD, and the length of the side is represented by $\mathrm{m}$ and $\mathrm{n}$, Then the formula (2) can be obtained from Equation (1):

$$
\begin{aligned}
& M=\frac{\mu_{0}}{4 \pi}\left(\iint \frac{d \vec{A}_{1} \bullet d \vec{A}_{2}}{R_{1}}+\iint \frac{d \vec{A}_{1} \bullet d \vec{C}_{2}}{R_{2}}+\iint \frac{d \vec{C}_{1} \bullet d \vec{A}_{2}}{R_{3}}+\iint \frac{d \vec{C}_{1} \bullet d \vec{C}_{2}}{R_{4}}\right. \\
& \left.+\iint \frac{d \vec{B}_{1} \bullet d \vec{B}_{2}}{R_{5}}+\iint \frac{d \vec{B}_{1} \bullet d \vec{D}_{2}}{R_{6}}+\iint \frac{d \vec{D}_{1} \bullet d \vec{B}_{2}}{R_{7}}+\iint \frac{d \vec{D}_{1} \bullet d \vec{D}_{2}}{R_{8}}\right)
\end{aligned}
$$
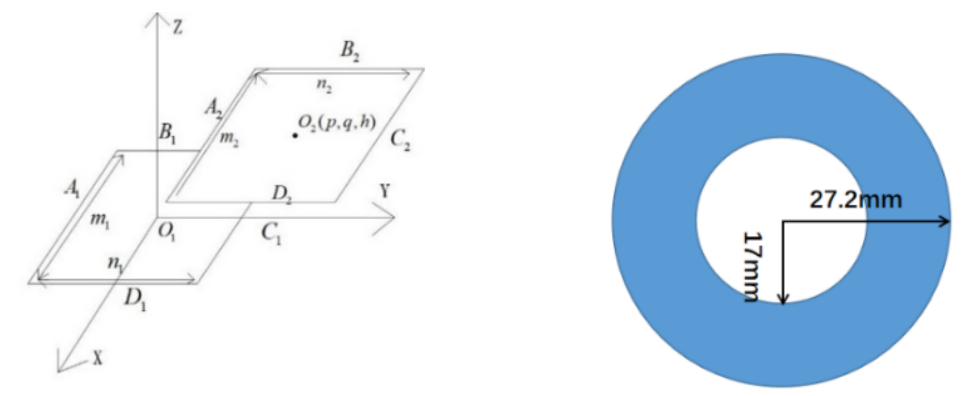

Figure 2. Rectangular coil electromagnetic model Figure 3. Rectangular coil electromagnetic model

After obtaining (2), we only need to calculate each term in (3) respectively to obtain the mutual inductance expression between the single-turn rectangular transmitting coil and the receiving coil. However, this expression is very complex and troublesome to calculate, so Maxwell electromagnetic simulation software is used for auxiliary calculation below.

\subsubsection{Coil Parameter Design}

In this paper, the wireless charging positioning of electric vehicles is obtained by detecting the inductive voltage of the detection coil, which directly determines whether the final positioning can be completed. Therefore, it is very important to design the parameters of the detection coil.

First on detecting coil wire material choice, for wireless transmission system, the size of the coil impedance has a great influence for the transmission efficiency, the actual situation should try to choose low resistance material testing coil, copper wire, generally is the first selection of conductor material, by the theory of electromagnetic field, the copper coil of the resistance by the loss of resistance and high frequency radiation resistance, expression is as follows:

$$
\begin{aligned}
& R_{0}=\sqrt{\frac{\omega \mu_{0}}{2 \sigma}} \frac{l}{2 \pi a}=\sqrt{\frac{\omega \mu_{0}}{2 \sigma}} \frac{N_{w} r}{a} \\
& R_{r}=\sqrt{\frac{\mu_{0}}{\varepsilon_{0}}}\left[\frac{\pi}{12} N_{W}^{2}\left(\frac{\omega r}{c}\right)^{4}+\frac{2}{3 \pi^{2}}\left(\frac{\omega h}{c}\right)^{2}\right]
\end{aligned}
$$

Where $\mu_{0}$ is the vacuum permeability, $\varepsilon_{0}$ is the vacuum dielectric constant, $\sigma$ is the coil conductivity, $N_{w}$ is the number of coil turns, and h is the coil turn spacing. $\mathrm{c}$ is the speed of light in vacuum, 1 is the length of the wire, and a is the radius of the wire section. For wireless charging systems for electric vehicles, the American Society of Automotive 
Engineers (SAE) has published a report on guidelines for wireless charging for electric vehicles SAE-J2954. The working frequency of electric vehicle wireless charging system is $85 \mathrm{kHz}$ [10], and the high-frequency radiation resistance in the general coil can be ignored.

Since the coupling coefficient of the circular coil is larger under the same conditions and can better reflect the voltage change after the receiving coil offset, the circular coil is selected as the detection coil for wireless charging positioning [11]. In other conditions are equal, the greater the area of the coil, the coil of the coupling coefficient will lead to greater, but this part of the design for electric vehicle charging positioning system in detecting coil, if is too big, can affect the positioning accuracy, so the coil area is not too big, so the preliminary design for detecting coil is composed of ring, outer ring is 27.2 $\mathrm{mm}$, inner ring is $17 \mathrm{~mm}$. As this experiment is an approximate qualitative analysis, although the number of coil turns will improve the overall accuracy, it will also make the simulation time too long. Therefore, copper ring is chosen to replace the multi-turn copper wire arrangement to simulate the detection coil. The final test coil design diagram and parameter table are shown in the following figure and table 1:

Table 1. detection coil design parameter sheet.

\begin{tabular}{cc}
\hline name of parameter & parameter \\
\hline shape & circular ring \\
texture & copper \\
external diameter & $27.2 \mathrm{~mm}$ \\
inner diameter & $17 \mathrm{~mm}$ \\
number of turns & 10 \\
\hline
\end{tabular}

\subsection{Analysis of Electromagnetic Model of Detection Coil}

In this paper, a positioning method based on three coils is proposed. Different shapes, sizes and inductance values of coils will affect their induced voltage. In order to reduce the interference of other variables on this study, three detection coils with the same shape, size and inductance values are selected, whose parameters are shown in Section 2.1.2.

One of the three coils, coil A, is placed on the straight line of the central axis of the rectangular coil, and the other two coils, coil B and coil $\mathrm{C}$, are placed around the two adjacent sides of the nearest edge of the rectangle and coil $\mathrm{A}$, and the three coils are placed in an equilateral triangle. The three detection coils are placed outside the receiving coil and at the same plane (same height) with the receiving coil. The magnetic field excited by the transmitting coil at a certain height is approximately circular in the XOY plane. Now, the origin is set at the position coincidant with the center of the transmitting coil, and the polar coordinate system is established. The mutual inductance is expressed as:

$$
M(r)=C
$$


In the above formula, $\mathrm{C}$ is a constant, indicating that the magnitude of mutual inductance is only related to the distance $\mathrm{r}$, and the parameter related to the center location of the coils on both sides is a monotone function $r$, if the center position of the receiving coil is at the point $\mathrm{P}(\mathrm{r} 1, \theta 1)$, without considering the case of the deflection of the coil Angle.

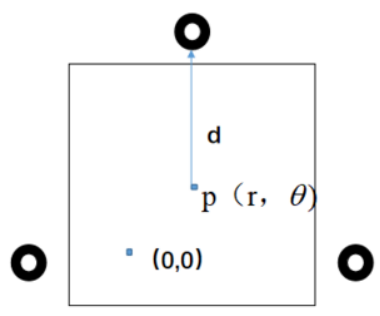

Figure 4. Schematic diagram of positioning system based on three detection coils.

Figure 4 shows the schematic diagram of positioning system based on three detection coils. $d$ is the distance between the three detection coils and the center of the receiving coil, and the three detection coils are arranged in an equilateral triangle. After the detection coil is positioned, the inductive voltage is proportional to the mutual inductance value, so the inductive voltage is only related to the distance between the coordinate plane and the detection coil, and it is a monotone function. If the distance between one point and three detection coils is a constant value in the plane, the positioning can be achieved with this method, and the proof is as follows:

Given a certain distance to any point $\mathrm{P}$ in the plane to form a circle with $\mathrm{P}$ as the center; An ellipse with $\mathrm{P}$ and $\mathrm{Q}$ as focal points is constituted by any point on the ellipse (which is not on the line PQ). If $\mathrm{M}$ is any point on the ellipse (which is not on the line $\mathrm{PQ}$ ), then there exists and only exists a symmetric $\mathrm{N}$ point on the line PQ, so that the distance between $\mathrm{P}(\mathrm{Q})$ and $\mathrm{M}$ and $\mathrm{N}$ is equal. Therefore, there is one or two points in the plane whose distance to any two known points is constant; In the equilateral triangle plane, if any point $M$ is taken, it can be found that the $M$ point can respectively find three points symmetric about the three sides, and these three points cannot coincide. Therefore, there exists and only one unique point on the plane whose distance to the three sides of the equilateral triangle is a fixed value.

What has been discussed above, there is no two different points at the same time make the detection coil voltage vector fully equal, so the reference point of threedimensional coordinate information and their corresponding detecting coil voltage information into a database, and then at the time of actual location, will be the voltage vector and reference voltage vector in the database, The approximate position of electric vehicle wireless charging receiving coil can be estimated by lookup table method. The specific exact position needs to be realized by a certain algorithm, which will be introduced below.

\section{Algorithm for Electric Vehicles Wireless Charging Positioning}

The second chapter studies the electric vehicle wireless energy transmission positioning system model based on three coils and the feasibility of three coils positioning method, this chapter will study the offset detection algorithm. 
It has been explained in Section 2.2 that when the system is working, the transmitting coil inputs excitation of a certain size to generate mutual inductance between the two coils, and inducted voltage is generated on the receiving coil side. The inducted voltage can be obtained by the following formula:

$$
u=\omega M I
$$

As can be seen from the above equation, when the current at the side of the transmitting coil remains constant, the mutual inductance value and the induced voltage value show a linear relationship. Therefore, the mutual inductance value of the detecting coil can be directly detected to realize the detection of the voltage value. The change of mutual inductance value has a great coupling relationship with the relative position of the transmitting coil and the receiving coil. Therefore, the offset of the coil position can be obtained through a certain coupling relationship only by detecting voltage.

In detecting migration cases before the induction voltage coil need to use wireless charging system between the two coil plane into a grid, and grid intersection set as the reference point, secure a receiving coil in each reference point three detecting coil induced voltage, when stored in three dimensions for $n * n$ numerical matrix, when the actual location, If the receiving coil is offset to a certain position, the proximity of the position to be measured to all the reference points can be described by the membership mathematical model in the fuzzy mathematical theory. So the algorithm of this paper is based on the membership mathematical model of fuzzy mathematics theory. By traversing the residual point voltage matrix obtained before, the four closest points can be found, and then the approximate range of the anchor point coordinates can be obtained. The following is a detailed introduction of the specific calculation method.

When the electric vehicle wireless charging positioning system is working, the three detection coils are kept at the same height and plane with the receiving coil, in an equilateral triangle distribution, and the relative position with the receiving coil is fixed. Firstly, the center point of the receiving coil is placed at each reference point, and the effective values of the three receiving coil voltages are recorded as $u_{p 1}, u_{p 2}, u_{p 3}$, and these values are stored in the matrix.

In practice, when the position of the receiving coil is offset, the voltage values of the three detection coils can be obtained. However, since the center point of the receiving coil cannot accurately fall on the reference point defined in this paper after every position offset, the accurate offset of the coil cannot be obtained. Instead, the proximity of the center of the receiving coil to several surrounding reference points after each position offset can be described. Set:

$$
\left\{\begin{array}{l}
u_{1}=u_{p 1}-u_{r 1} \\
u_{2}=u_{p 2}-u_{r 2} \\
u_{3}=u_{p 3}-u_{r 3}
\end{array}\right.
$$

Where, $u_{r}$ is the voltage value of the grid point measured previously, namely the reference point; $u_{p}$ is the inductive voltage of the detection coil measured after the actual position of the receiving coil is offset; $\Delta \mathrm{U}$ is used to describe the proximity of the center point of the receiving coil to the reference point of meshing, which is described by membership function in this paper. According to the previous analysis in Section 2.2, the smaller $\Delta \mathrm{U}$ is, the closer the center point of the receiving coil is to the sub-meshed reference point. In this paper, a membership function model with small distribution is selected. $\delta$ can be defined by the membership function of normal distribution: 


$$
\delta=e^{-a \Delta U^{2}}
$$

After obtaining the voltages of the three detection coils at a certain position, the membership function is used to obtain the $\delta$ of each reference point, thus an $n * n$ matrix can be obtained:

$$
\Delta=\left[\begin{array}{ccccc}
\delta_{11} & \cdot & \cdot & \cdot & \delta_{1 \mathrm{n}} \\
\cdot & \cdot & & & \\
\cdot & & \cdot & & \\
\cdot & & & \cdot & \\
\delta_{\mathrm{n} 1} & & & & \delta_{\mathrm{nn}}
\end{array}\right]
$$

Due to $\delta$ describes specific with all the grid reference point proximity, therefore, its size and specific only related to the distance between the reference point, the reason for out of four is the maximum and the nearest grid reference point, specific ergodic matrix of $\mathrm{n} * \mathrm{n}$ elements, can get $\delta$ values of the four largest elements, write them respectively for the $\delta_{A}, \delta_{B}, \delta_{C}, \delta_{D}$, These four points, theoretically represent the four meshing reference points closest to the center point of the receiving coil.

Since the meshing reference points were originally designed, the coordinates of points $\mathrm{A}, \mathrm{B}, \mathrm{C}$ and $\mathrm{D}$ are known, at this point, the coordinate of the center position of the receiving coil can be obtained from the coordinates of the four meshing reference points and their proximity $\delta$, and the calculation formula is:

$$
\left\{\begin{array}{l}
x=\frac{x_{a} \delta_{a}+x_{b} \delta_{b}+x_{c} \delta_{c}+x_{d} \delta_{d}}{\delta_{a}+\delta_{b}+\delta_{c}+\delta_{d}} \\
y=\frac{y_{a} \delta_{a}+y_{b} \delta_{b}+y_{c} \delta_{c}+y_{d} \delta_{d}}{\delta_{a}+\delta_{b}+\delta_{c}+\delta_{d}}
\end{array}\right.
$$

The obtained $\mathrm{x}$ and $\mathrm{y}$ are respectively the abscissa and ordinate of the center point of the receiving coil to be solved, so as to realize the positioning of the electric vehicle wireless charging coil. In the following chapters, the algorithm will be used to locate the positioning coil in the wireless charging system of electric vehicles, and the accuracy of the algorithm will be analyzed.

\section{Simulation and Experimental Verification of Electric Vehicle Wireless Charging Positioning System}

According to the analysis in the previous chapter, for the wireless charging positioning system of electric vehicles, if the current of the transmitting coil and the system frequency are constant, then the position change of the receiving coil can be represented by the voltage change of the three detection coils through a certain algorithm to realize the positioning of the coil of the wireless transmission system of electric vehicles.

In this chapter, relevant simulation, experiment and error analysis will be carried out for the positioning system of electric vehicle wireless energy transmission coil under the condition of asynchronous length, and the positioning accuracy and error sources will be analyzed. 


\subsection{Simulation Results of Three Detection Coil Positioning System}

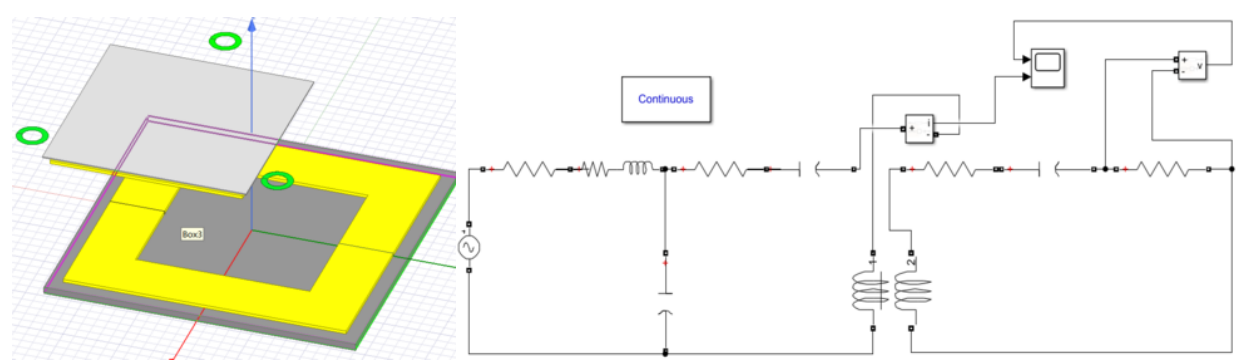

Figure 5. Electromagnetic model of electric vehicle wireless charging. Figure 6. LCC-S circuit simulation model.

As shown in Figure 5, the wireless charging positioning system of electric vehicles consists of transmitting coil, receiving coil and three detection coils of exactly the same size and shape. The center point of the transmitting coil in the figure is fixed at the origin of coordinates, and the side length of the square transmitting coil is $50 \mathrm{~cm}$. The position of the receiving coil can be moved in the same height plane. The side length of the square receiving coil is $30 \mathrm{~cm}$ and the height is $17.54 \mathrm{~cm}$. The parameters of the detecting coil are mentioned in Section 2.1.2.

In the model established in this paper, the size of the transmitting coil is designed to be $50 \mathrm{~cm} * 50 \mathrm{~cm}$, the number of turns is 10 , and the current is $24.9 \mathrm{~A}$. The receiving coil is $30 \mathrm{~cm} * 30 \mathrm{~cm}$, and the number of turns is 10 . The detection coil and the receiving coil are in a height plane, and the three detection coils are arranged around the receiving coil in an equilateral triangle.

Firstly, a region in the receiving coil plane is gridded and $n * n$ reference points are obtained. In this paper, the size of this region is designed as $35 * 35 \mathrm{~cm}$. Three groups of simulations are carried out, and the step sizes are set as $2 \mathrm{~cm}, 3 \mathrm{~cm}$, and $4 \mathrm{~cm}$ respectively, that is, the distance between two adjacent gridded reference points is $2 \mathrm{~cm}, 3 \mathrm{~cm}$, and $4 \mathrm{~cm}$. Then the model was simulated, and the step size was set to $1 \mathrm{~cm}$ during the simulation, that is, a grid of $35^{*} 35$ could be obtained. Data processing was done on this grid, that is, one of every or several points was taken as the reference point to obtain three groups of data, which were stored in three tables. They respectively represent the mutual inductance between the three detection coils and the transmitting coil when the center point of the receiving coil is located at each grid reference point

The algorithm of this paper is by detecting coil and the transmitter coil between the induced voltage to achieve localization work, therefore need to convert the mutual inductance existing data into data induction voltage, the current value can be built with the help of the LCC-S model simulation in MATLAB, the circuit model is as shown in figure 6 .

In the simulation model of LCC-S circuit, the peak value of the AC power supply is $380 \mathrm{~V}$, the frequency is $85 \mathrm{kHz}$, the series resistance of the primary side is $0.2 \Omega$, the inductance is $0.02 \mathrm{mH}$, the shunt capacitance of the primary side of the circuit is $0.175 \mu \mathrm{F}$, and the series capacitance of the secondary side is $0.0377 \mu \mathrm{F}$. At this point, the current value I of the circuit flowing through the primary coil can be obtained. Using the formula:

$$
\mathrm{u}=2 \pi \times 85000 \times 32 \times M \div \sqrt{2}
$$


The three groups of data obtained previously were calculated according to the above formula, and the induced voltage of the three detection coils when the center point of the receiving coil is located at each meshing reference point could be obtained. They were stored in the three matrices to facilitate positioning calculation by using the algorithm designed. The simulation results are shown in Table 2.

The experimental device is shown in the figure 7 , and it can be concluded that the algorithm in this paper can have small errors in both simulation and experiment.

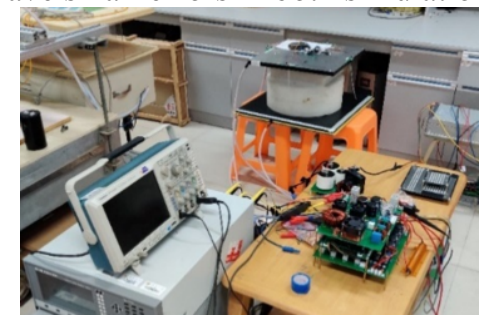

Figure 7. Electric vehicle wireless charging positioning system experimental device diagram.

Table 2. Ten groups of data with step sizes of $2 \mathrm{~cm}, 3 \mathrm{~cm}$ and $4 \mathrm{~cm}$.

\begin{tabular}{|c|c|c|c|}
\hline $\begin{array}{l}\text { The simulation } \\
\text { serial number }\end{array}$ & $\begin{array}{l}\text { The theoretical position } \\
\text { in } 18 * 18 \text { grid }\end{array}$ & $\begin{array}{l}\text { The actual position in } \\
18 * 18 \text { grid }\end{array}$ & $\begin{array}{l}\text { Error } \\
(\mathrm{cm})\end{array}$ \\
\hline 1 & $(1.5,4)$ & $(1.49,4)$ & 0.1 \\
\hline 2 & $(7.5,7)$ & $(7.55,6.9)$ & 0.13 \\
\hline 3 & $(5.5,10.5)$ & $(5.78,10.41)$ & 0.57 \\
\hline 4 & $(2,16)$ & $(2.02,16.03)$ & 0.05 \\
\hline 5 & $(17.5,8)$ & $(17.10 .7 .61)$ & 1.12 \\
\hline 6 & $(2.5,10.5)$ & $(2.45,10.66)$ & 0.34 \\
\hline 7 & $(10.5,7.5)$ & $(10.61,7.35)$ & 0.37 \\
\hline 8 & $(8.5,17.5)$ & $(9.10,17.45)$ & 1.2 \\
\hline 9 & $(9,2)$ & $(8.82,2.39)$ & 0.86 \\
\hline 10 & $(7.5,5.5)$ & $(7.58,5.08)$ & 0.86 \\
\hline $\begin{array}{l}\text { The simulation } \\
\text { serial number }\end{array}$ & $\begin{array}{l}\text { The theoretical position } \\
\text { in } 12 * 12 \text { grid }\end{array}$ & $\begin{array}{l}\text { The actual position in } \\
12 * 12 \text { grid }\end{array}$ & $\begin{array}{l}\text { Error } \\
(\mathrm{cm})\end{array}$ \\
\hline 1 & $(1.33,3)$ & $(1.23,2.83)$ & 0.59 \\
\hline 2 & $(5.33,5)$ & $(5.1,4.98)$ & 0.69 \\
\hline 3 & $(4,7.33)$ & $(4.52,7.11)$ & 1.69 \\
\hline 4 & $(1.67,11)$ & $(1.83,11.1)$ & 0.57 \\
\hline 5 & $(12,5.67)$ & $(11.53,5.42)$ & 1.6 \\
\hline 6 & $(2,7.33)$ & $(1.85,7.3)$ & 0.46 \\
\hline 7 & $(7.33,5.33)$ & $(7.05,5)$ & 1.3 \\
\hline 8 & $(6,12)$ & $(6.03,12)$ & 0.09 \\
\hline 9 & $(6.33,1.67)$ & $(6.19,2.02)$ & 1.13 \\
\hline 10 & $(5.33,4)$ & $(5.34,3.59)$ & 1.23 \\
\hline $\begin{array}{l}\text { The simulation } \\
\text { serial number }\end{array}$ & $\begin{array}{l}\text { The theoretical position } \\
\text { in } 9^{*} 9 \text { grid }\end{array}$ & $\begin{array}{l}\text { The actual position in } \\
9 * 9 \text { grid }\end{array}$ & $\begin{array}{l}\text { Error } \\
(\mathrm{cm})\end{array}$ \\
\hline 1 & $(1.25,2.5)$ & $(1.23,2.68)$ & 0.72 \\
\hline 2 & $(4.25,4)$ & $(4,4)$ & 1 \\
\hline 3 & $(3.25,5.75)$ & $(3,6)$ & 1.41 \\
\hline 4 & $(1.5,8.5)$ & $(2.17,7.83)$ & 3.82 \\
\hline 5 & $(9.25,4.5)$ & $(8.98,4)$ & 2.27 \\
\hline 6 & $(1.75,5.75)$ & $(2.95,5.03)$ & 5.6 \\
\hline
\end{tabular}




\begin{tabular}{cccc}
7 & $(5.75,4.25)$ & $(6,4)$ & 1.41 \\
8 & $(4.75,9.25)$ & $(5.51,9)$ & 3.2 \\
9 & $(5,1.5)$ & $(4.96,1.57)$ & 0.32 \\
10 & $(4.25,3.25)$ & $(4,3.23)$ & 1 \\
\hline
\end{tabular}

\subsection{Error Analysis of Simulation Results of Detection Coil Positioning System}

This paper simulates the situation that the receiving coil is located in ten different positions. That is, 10 serial numbers are input into Visual $\mathrm{C}++$ to simulate the real position of the receiving coil in the electric vehicle wireless energy transmission system, and the theoretical and actual positions of ten groups of data can be obtained in $18 * 18$, $12 * 12$ and $9 * 9$ grids respectively. Their positioning error sizes were analyzed and compared, and four ranges with error sizes of less than or equal to $1 \mathrm{~cm}, 1 \mathrm{~cm}-2 \mathrm{~cm}, 2 \mathrm{~cm}-$ $3 \mathrm{~cm}$ and greater than $3 \mathrm{~cm}$ were selected respectively. The results are shown in Table 3.

Table 3. Simulation error statistics under asynchronous length.

\begin{tabular}{lcccc}
\hline error magnitude & less than or equal to $1 \mathrm{~cm}$ & $1 \mathrm{~cm}-2 \mathrm{~cm}$ & $2 \mathrm{~cm}-3 \mathrm{~cm}$ & greater than \\
\hline step length $2 \mathrm{~cm}$ & 8 & 2 & 0 & 0 \\
step length $3 \mathrm{~cm}$ & 5 & 5 & 0 & 0 \\
step length $4 \mathrm{~cm}$ & 4 & 2 & 1 & 3 \\
\hline
\end{tabular}

As can be seen from Table 3, when the step size is $2 \mathrm{~cm}$, the errors of the ten groups of tests are all within $2 \mathrm{~cm}$, and the accuracy of this algorithm reaches the expected standard. The experiment in this paper was only carried out when the step size was $2 \mathrm{~cm}$, and the results showed that the ten groups of data were all within the allowable error range. It can be seen that both simulation and experiment can verify the correctness of the algorithm in this paper. Therefore, this algorithm is feasible. When the step size becomes larger, the positioning error will become larger. The following paragraphs will speculate on the error sources of the algorithm.

By analyzing the algorithm, electromagnetic model and data designed in this paper, it can be concluded that the reasons for errors are as follows:

(1) The algorithm in this paper is established based on the membership mathematical model in fuzzy mathematics theory. $\delta$ represents the degree of proximity between the point to be solved and the gridded reference point, which is an inaccurate value. Therefore, the coordinate calculated by multiplying the reference point by their respective degree of proximity is also inaccurate and may cause errors.

(2) In the electromagnetic model of the wireless charging positioning system of electric vehicles in this paper, the inductive voltage generated by the excitation of the transmitting coil in the three detection coils differs greatly, which may be related to the placement of the three detection coils. In the final calculation, the three groups of data were normalized. That is, the three groups of data are changed into three groups of approximately equal size through scaling and scaling. Although scaling and scaling do not change the magnitude relationship of the original induced voltages at several grid reference points, the accuracy will therefore change. So the error is going to go up.

To sum up, this paper proposes and implements a three-coil based wireless charging positioning algorithm for electric vehicles. The inductive voltage data are processed by the membership degree mathematical model in fuzzy mathematics theory, and the feasibility of the scheme is verified by simulation. 


\section{Conclusion}

Aiming at the requirement of deviation degree of electric vehicle wireless charging coil, this paper designed a positioning algorithm of electric vehicle wireless charging based on three coils. This algorithm was designed based on the membership mathematical model in fuzzy mathematics theory. In this paper, theoretical analysis, simulation analysis and experiment are carried out on the electric vehicle wireless charging system model, and error analysis is carried out on the simulation results. When the step size is $2 \mathrm{~cm}$, the errors of ten groups of tests are all within $2 \mathrm{~cm}$. The accuracy of this algorithm reaches the expected standard, and the algorithm meets the working requirements of the system, and the scheme is feasible.

\section{References}

[1] Marketsand-Markets. Wireless Charging Market Analysis and Forecast by Technology, Range, Components (Transmitter and Receiver for Various Devices), Uses (Appliances, Defense, Medical, Automotive, Industrial), and Region. [J].2014

[2] Wu J, Liu X, Brief analysis of electric vehicle charging technology and electric change technology[J]. Automobile practical technology, 2021,46(12):8-10.

[3] Wireless Power Transfer for Light-Duty Plug-In/Electric Vehicles and Alignment Methodology, Standard SAEJ2954, May 2016. [online]. Available: http://standards.sae.org/j2954_20120122.html.

[4] Birrell S A, Wilson D, Yang C P, et al. How driver behaviour and parking alignment affects inductive charging systems for electric vehicles $[\mathrm{J}]$. Transportation Research Part C, 2015, 58:721-731.

[5] Yan M S, Research on Location Technology of Electric Vehicle Wireless Charging System Based on Magnetic Field Induction[D]. Bejing: University of Chinese Academy of Sciences, 2014.

[6] Zhao J B, Cai T, Duan S X, et al. Relay method suitable for piecewise dynamic wireless charging[J]. Automation of Electric Systems. 2016, 40(16): 64-70.

[7] Cheng Z Q, Chen H, Wu J D, HE X N. An online wireless charging and positioning system for electric vehicles based on differential inductance $[\mathrm{P}]$. Zhejiang Province: CN109895643A,2019-06-18.

[8] Fang Q Q. Research on Dynamic Wireless Power Supply System Control of Electric Vehicle Based on Magnetic Positioning Detection Technology[D]. Bejing: Beijing Jiaotong University, 2017, 18-53.

[9] Allan L, Mohammad N. Wireless Charging System Localization for Electric Vehicles Using RSSI[J]. SAE International Journal of Passenger Cars - Electronic and Electrical Systems, 2015, 8(2):2015-01-0283

[10] http:/www.sae.org/servlets/pressRoom?OBJECT_TYPE=PressReleases\&PAGE= showRelease\&RELEAS.

[11] Zhai Z, Sun Y, Dai X, et al. Modeling and analysis of magnetic resonance mode radio energy transmission system[J]. Proceedings of the CSEE, 2013,32(12):155160 . 